\title{
Quantifying online visuomotor feedback utilization in the frequency domain
}

\author{
John de Grosbois ${ }^{1,2} \cdot$ Luc Tremblay ${ }^{1,2}$
}

Published online: 5 November 2015

(C) Psychonomic Society, Inc. 2015

\begin{abstract}
The utilization of sensory information during activities of daily living is ubiquitous both prior to and during movements (i.e., related to planning and online control, respectively). Because of the overlapping nature of online corrective processes, the quantification of feedback utilization has proven difficult. In the present study, we primarily sought to evaluate the utility of a novel analysis in the frequency domain for identifying visuomotor feedback utilization (i.e., online control). A second goal was to compare the sensitivity of the frequency analysis to that of currently utilized measures of online control. Participants completed reaching movements to targets located 27,30 , and $33 \mathrm{~cm}$ from a start position. During these reaches, vision of the environment was either provided or withheld. Performance was assessed across contemporary measures of online control. For the novel frequency analysis presented in this study, the acceleration profiles of reaching movements were detrended with a 5 th-order polynomial fit, and the proportional power spectra were computed from the residuals of these fits. The results indicated that the use of visual feedback during reaching movements increased the contribution of the $4.68-\mathrm{Hz}$ frequency to the residuals of the acceleration profiles. Comparisons across all measures of online control showed that the most sensitive measure was the squared Fisher transform of the correlation between the positions at $75 \%$ and $100 \%$ of the movement time. However, because such correlational measures can be contaminated by
\end{abstract}

Luc Tremblay

luc.tremblay@utoronto.ca

1 Faculty of Kinesiology and Physical Education, University of Toronto, Toronto, Ontario, Canada

2 Perceptual Motor Behavior Laboratory, University of Toronto, 55 Harbord Street, Toronto, Ontario M5S 2W6, Canada offline control processes, the frequency-domain analysis proposed herein represents a viable and promising alternative to detect changes in online feedback utilization.

Keywords Online control $\cdot$ Discrete reaching $\cdot$ Power spectra $\cdot$ Visuomotor feedback

The human sensorimotor system must be sensitive to the dynamic nature of the environment in which voluntary movements are completed. One source of sensory information that is obviously important for the maintenance of movement accuracy is visual information. This capacity is exemplified through the use of visual feedback during an ongoing movement (i.e., online control). For example, the use of feedbackrelated processes is apparent through the changes in accuracy brought about by manipulations of visual information after movement onset (e.g., Elliott \& Madalena, 1987; Heath, 2005; Keele \& Posner, 1968; Woodworth, 1899; Zelaznik, Hawkins, \& Kisselburgh, 1983). Although the influence of online feedback-based processes is evident in behavioral outcomes, the quantification of online feedback utilization and the manner in which feedback is utilized have been the subjects of much debate (e.g., Elliott \& Hansen, 2010; Khan et al., 2006).

Models of online control have been proposed to explain sensory feedback utilization during action, and many researchers have worked from the general assumption that accuracy is maintained via a servo-mechanism (i.e., Craik, 1947). For example, to explain the use of online feedback during action, Crossman and Goodeve (1963/1983) proposed a model of visual control wherein visual corrective feedback processes occur in an iterative fashion whereby corrections are completed serially at a fixed latency. Likewise, Howarth, Beggs, and Bowden (1971) also argued that feedback 
processes require a fixed latency of time from which to act. This latency, however, would be the limiting factor regarding the number of corrections per movement. More recently, Meyer, Abrams, Kornblum, Wright, and Smith (1988) argued that the timing of corrective submovements could instead be described by statistically optimal processes. That is, corrections are completed at a point in the movement at which they would optimally improve movement accuracy. Although differences existed between the above models, common threads between them have included the notions that online feedback utilization is likely an iterative process that requires time.

The models described above also relied primarily on the idea that discrete discontinuities in kinematic profiles could be interpreted as evidence for online feedback utilization. As such, methodologies developed for the measurement of online feedback utilization have often relied on the presence of discrete trajectory discontinuities. For example, Khan, Franks, and Goodman (1998) developed an index of error correction efficiency based on reduction in the errors associated with a secondary submovement (i.e., the error correction phase). Such submovements are often identified through an analysis of trajectory discontinuities (see Walker, Philbin, Worden, \& Smelcer, 1997). Subsequent findings, however, have provided evidence that the simple reliance on the identification of trajectory discontinuities may not always provide conclusive evidence regarding feedback utilization. For instance, Elliott, Carson, Goodman, and Chua (1991) argued that discrete discontinuities in kinematic profiles may not always be apparent. That is, online corrective processes may instead occur in an overlapping manner, such that individual corrective submovements may not be discernible at the kinematic level. One source of evidence for this assertion came from the finding that the number of discrete discontinuities in a trajectory does not systematically decrease with the withdrawal of vision of the environment (e.g., Elliott et al., 1991). In comparison, the time spent moving following peak limb velocity (i.e., the deceleration phase duration) has been reported to be more tightly linked to the vision manipulations employed. Therefore, it was argued that online corrective processes likely occurred in a temporally overlapping fashion that resulted in smooth trajectory profiles. This idea has become known as the "pseudocontinuous control model." The existence of overlapping corrections poses a potential problem for the identification and quantification of online feedback utilization during movement, especially for techniques that rely on discrete discontinuities in the movement trajectory.

Another finding incongruent with the quantification of online feedback utilization on the basis of the number of discrete discontinuities in a trajectory was presented by Dounskaia, Wisleder, and Johnson (2005). They examined reciprocal and discrete reaching movements and evaluated the numbers of discontinuities in the profiles. In the presence of online visual information, more discontinuities were observed for the trajectories of the discrete than of the reciprocal movements. Thus, it was argued that some discontinuities may come about simply through the process of slowing a limb to a stop rather than as a consequence of online feedback utilization. If it is often not apparent that a correction has been made, nor that the presence of discontinuities can be associated with online feedback utilization, how can one accurately assess the relative use of corrective processes on the basis of discontinuities alone?

To address the above issue, many kinematic measures have been proposed as proxies for the quantification of the involvement of feedback-related processes for the online control of voluntary movements. That is, in the absence of the ability to directly measure and quantify online feedback utilization at the neurophysiological level, many behavioral measures have been put forth to provide best estimates regarding the influence of online visuomotor mechanisms. These include the time after peak velocity (e.g., Elliott et al., 1991), trial-totrial variability (e.g., Khan et al., 2003; Messier \& Kalaska, 1999), measurements of the higher derivatives of position with respect to time, such as jerk (e.g., Flash \& Hogan, 1985), and correlations of position at different movement proportions (e.g., Fisher ${ }^{2}$, proposed by Bernier, Chua, Franks, \& Khan, 2006; $R^{2}$, described by Heath, 2005; Heath, Westwood, \& Binsted 2004; $r$, from Messier \& Kalaska, 1999; and Fisher's $Z$ transformation of the correlation between the distances traveled to and after peak velocity, put forward by Elliott, Binsted, \& Heath, 1999). Increases in the time after peak velocity have been proposed to be associated with online feedback utilization in part because it was initially believed that the early phases of movement were completed without the influence of feedback (e.g., Woodworth, 1899). Thus, greater reliance on feedback would extend to the later portion of the movement. Alternatively, the analysis of normalized jerk (i.e., the third derivative of position) across a movement (i.e., a "jerk score"; e.g., Tresilian, Stelmach, \& Adler, 1997) has been proposed as a proxy for online feedback utilization. Movements unaltered by feedback have been assumed to be planned in a manner that minimizes jerk (i.e., discontinuities), and thus, increases in the normalized jerk indicated a greater contribution of feedback utilization to the performance (e.g., Flash \& Hogan, 1985). More recent methodologies for the quantification of online feedback utilization during reaches have relied on between-trial variability and correlations (e.g., Messier \& Kalaska, 1999). In general, movements unaltered by online feedback have been assumed to result in relatively stereotyped trajectories across a number of trials. Therefore, the predictability of position between different proportions of a trajectory theoretically could be utilized to assess the influence of feedback-related processes. Lower correlations have therefore been taken as evidence for adjustments across trajectories, which have been attributed to online feedback utilization (e.g., Elliott et al., 1999; Heath, 2005). Although each 
of these measures can provide evidence supporting the possibility that feedback mechanisms have been utilized, all are potentially contaminated by offline (i.e., trial-to-trial) changes in behavior. Indeed, changes in the stereotypedness of the profile can also come about through changes in the planning process (e.g., Plamondon \& Alimi, 1997). Evidence for this assertion has come from the finding that performance is maximized when the availability of visual feedback is scheduled in a blocked manner, as compared to a randomized schedule (i.e., Elliott \& Allard, 1985). In addition, both the relative certainty of the availability of feedback (i.e., Zelaznik et al., 1983) and the availability of vision on preceding trials (i.e., Cheng, Luis, \& Tremblay, 2008) have been shown to influence endpoint accuracy across reaches. Thus, changes in reaching performance, which could otherwise be erroneously attributed to online feedback utilization, could theoretically come about through offline changes in performance.

Another potential solution to the problems associated with the measurement of online control resides in the frequency domain. The relative contributions of different oscillation frequencies to a signal can be represented by a normalized power spectral density function (see, e.g., Warner, 1998). These frequencies can also be represented as latencies (i.e., seconds per cycle, rather than cycles per second). Because corrective reaction times to visual perturbations tend to be relatively stable throughout reaching movements (e.g., Cressman, Cameron, Lam, Franks, \& Chua, 2010; Proteau, Roujoula, \& Messier, 2009; Saunders \& Knill, 2003), the influence of online corrective feedback processes based on visual information likely exerts its influence at a specific frequency (i.e., a latency). Thus, rather than simply estimating the number of discontinuities in a trajectory, the contributions of specific iterative influences, presumably associated with specific feedback processes, can be quantified and evaluated. Additionally, representing the trajectory in the frequency domain circumvents the difficulty of identifying online feedback utilization caused by temporally overlapping corrective processes. These overlapping processes contribute to the same frequencies in the frequency domain (i.e., they are additive). This type of analysis has been previously applied to a line-drawing task (e.g., Van Galen, Van Doorn, \& Schomaker, 1990), small goal-directed finger and wrist movements (van Galen \& Schomaker, 1992), and reciprocal reaching trajectories (e.g., Van Donkelaar \& Franks, 1991). However, these studies needed be extended by including comparisons of performance across different sensory environments in order to more directly assess online control processes.

One goal of the present experiment was to directly test the prediction that differences in performance due to online feedback utilization would be measurable in the frequency domain (i.e., the proportional power spectra) of the limb trajectories of discrete reaching movements. Participants were tasked with completing goal-directed reaching movements to stationary targets in the presence or absence of online visual feedback. These two sensory environments were chosen to identify the frequencies accounting for the differences in performance mediated by visual feedback processes during reaching movements. Due to the wave-like form of the acceleration traces of discrete reaching movements (i.e., a sine-wave shape), acceleration traces were chosen for the frequency analysis. Because the process of differentiation amplifies highfrequency noise in signals (e.g., Bracewell, 1986), acceleration traces were gathered directly using a triple-axis accelerometer, rather than from double-differentiated position data. Kinematic profiles were converted to a frequency representation using a fast Fourier transform (FFT). The FFT output was then converted to a proportional power spectrum (pPower; \% spectral power), so that differences between the two visual conditions could be assessed directly (see Warner, 1998). We hypothesized that increased feedback utilization in the presence of online visual information would result in an increase in the pPower as compared to performance in the absence of online visual feedback. The specific frequency at which this difference resided could potentially identify the iterative rate at which visual feedback has been utilized. This frequency was hypothesized to be in the range of up to approximately $10 \mathrm{~Hz}$ (i.e., iterative rates of feedback utilization up to around 100 ms; e.g., Carlton, 1992; Saunders \& Knill, 2003; Zelaznik et al., 1983). Although separate online control processes have been proposed (i.e., impulse vs. limb-target regulation processes; see Elliott et al., 2010), the explicit quantification of these subprocesses was deemed to be beyond the scope of the present study.

A secondary goal of the present experiment was to compare the herein-proposed frequency analysis to contemporary measures of online control with regard to their abilities to identify differences in performance between conditions with and without online vision available (see also Elliott \& Hansen, 2010; Khan et al., 2006). This was achieved by examining the magnitudes of the effect sizes of significant effects involving the visual availability manipulations. In addition to the effect sizes associated with these significant effects, the effect sizes associated with theoretically driven individual comparisons were also investigated. This would allow for the identification of the individual comparisons associated with particular measures of online control that were most sensitive to changes in the availability of visual information.

\section{Method}

Ten participants (three female seven male; mean age $=$ 25 years, $S D=5.8$ ) were recruited for participation from the University of Toronto community. All participants were selfdeclared right-handed and had normal or corrected-to-normal vision at the time of participation. All participants gave 
informed written consent prior to participation and were reimbursed (\$10 CAD).

Participants were seated in front of a custom aiming console. A palpable home position was located $30 \mathrm{~cm}$ to the right of the participant's midline. Participants wore an infrared light-emitting diode (LED) on their right index finger. Their index finger position was then monitored at a sampling rate of $200 \mathrm{~Hz}$ by an Optotrak Certus motion-tracking system (Northern Digital, Waterloo, ON, CA). In addition, participants wore an orthotic wrist brace and held a flat, rigid custom plastic tool $(2 \mathrm{~cm} \times 12 \mathrm{~cm} \times 0.4 \mathrm{~cm})$ against the underside (i.e., the inferior surface) of their right index finger (i.e., it held the finger straight). This tool was held such that it extended from approximately the base of the participants' index finger to the base of the distal phalanx. Consequently, the pad of the participant's index finger was not obstructed. A triple-axis accelerometer was affixed to this tool (Model MMA7361L, Sparkfun Electronics, Niwot, CO, USA), and the accelerometer sampled concurrently with the Optotrak data at $200 \mathrm{~Hz}$ using the analog-input ports on a multifunction data acquisition card (Model PCI-6042E; National Instruments, Austin, TX, USA). Participants were instructed to complete a rightto-left (i.e., lateral-to-medial) goal-directed reaching movement from the home position to a target LED (i.e., upon the target's illumination) as quickly and accurately as possible, but with a greater emphasis on movement accuracy. Participants were also instructed to keep the accelerometer (i.e., the moving finger/tool) as close to perpendicular to the axis of movement as was comfortably possible during reaches. The direction of movement was chosen primarily for two reasons. First, ending the movements near the participant's midline minimized the potential for occlusion of the target by the reaching limb. Second, movements along the medial-lateral axis were chosen to minimize cross-contamination of the axes of the accelerometer. Three target amplitudes were used (27, 30 , and $33 \mathrm{~cm}$ ), and the $30-\mathrm{cm}$ target amplitude LED was positioned approximately at the participant's midline (i.e., mid-sagittal plane). All target LEDs were situated beneath the surface of the aiming console and were not visible to the participant until they were illuminated. Three target amplitudes were employed in an attempt to reduce the predictability of the specifics of an upcoming trial and, thus, in turn to reduce the degree to which movements could be influenced by offline processing. The task itself - namely a simple discrete reaching movement-was chosen to provide a straightforward context from which to infer changes in performance due to online feedback utilization, while also being consistent with the literature that has investigated online control mechanisms (e.g., Elliott \& Hansen, 2010; Plamondon \& Alimi, 1997; Wallace \& Newell, 1983).

Reaching movements were completed in two sensory environments: with online visual feedback (i.e., full-vision condition: FV) and without such feedback (i.e., no-vision condition: NV). The availability of online visual information was manipulated using liquid crystal goggles (Translucent Technologies, Toronto, ON, Canada). In the NV condition, the goggles occluded visual information at movement onset. The movement onset was determined by the first time that a velocity threshold of $30 \mathrm{~mm} / \mathrm{s}$ was achieved in the primary movement axis for two consecutive samples. A movement's end was determined by the first time after which the finger velocity in the primary movement axis fell below $30 \mathrm{~mm} / \mathrm{s}$ for two consecutive samples. Participants completed ten familiarization trials, followed by a total of $30 \mathrm{FV}$ trials and $30 \mathrm{NV}$ trials to each of the three targets. A total of 180 experimental trials were thus completed by each participant (i.e., 30 Trials $\times$ 2 Vision Condition $\times 3$ Target Amplitudes) in approximately $30 \mathrm{~min}$. The order of the trials of both vision conditions was randomized within the experiment, and the experimental procedures and data acquisition were controlled via custom MATLAB scripts (The Mathworks Inc, Natick, MA, USA).

\section{Design and analysis}

Participants' performance was quantified and analyzed across five sets of dependent variables. The first four of these included a selection of currently utilized measures for the quantification of online control, and the fifth was the novel frequencybased approach forwarded in the present study. The currently utilized methodologies considered included both within-trial and between-trial measures of online control whereas the frequency-based approach employed was a within-trial measure. The currently utilized methodologies were first evaluated in order to validate performance differences across the vision conditions prior to the forthcoming analysis in the frequency domain.

The first set of conventional dependent variables were calculated with respect to movement end and were operationally defined as movement end kinematic measures. These included temporal dependent variables such as movement time (MT), time to peak velocity (TTPV), time after peak velocity (TAPV), and proportional time after peak velocity (i.e., pTAPV). MT was defined as the elapsed time between movement onset and movement end. The magnitude and timing of peak velocity was determined by the maximal velocity achieved between movement onset and movement end. Also, endpoint spatial measures were employed, including constant error (i.e., endpoint bias: CE) and variable error (i.e., endpoint standard deviation: VE). Each of these variables was quantified in both the primary and secondary movement axes (e.g., $\mathrm{CE}_{\text {Prim }}$ and $\mathrm{CE}_{\mathrm{Sec}}$ represent the constant errors in the primary and secondary axes, respectively). The second set was spatio-temporal in nature and included only the peak velocity in the primary movement axis (i.e., PV). The third set of dependent variables were based on trajectory characteristics (i.e., trajectory-based kinematic measures), and included jerk 
score (i.e., JScore) as well as variability analyses at $25 \%, 50 \%$, $75 \%$, and $100 \%$ of the MT (e.g., Variability @ $75 \%$ MT). The JScore was calculated via the following equation:

JScore $=\sqrt{\left(\frac{S S_{\text {Jerk }}}{2} \times\left[\left(M T_{\text {Seconds }}\right)^{5} /\left(\frac{\text { Jerk }_{\text {Volts Range }}}{1000}\right)^{2}\right]\right)}$,

where $\mathrm{SS}_{\text {Jerk }}$ represents the sum of the squared jerk across the trajectory, $\mathrm{MT}_{\text {Seconds }}$ represents the movement time in seconds, and Jerk VoltsRange $_{\text {represents the difference between the maximum }}$ and minimum jerks recorded from the differentiated accelerometer trace, across a trajectory. The variability analysis was associated with the raw spatial variability (i.e., the between-trial within condition standard deviation) at different movement proportions, across all trials within a condition. Additionally, betweentrial correlational analyses were completed at 25,50 , and $75 \%$ of MT. These analyses included: $r$-squared analysis (i.e., $R^{2}$ ), Fisher $Z$ analysis (i.e., Fisher), and Fisher $Z$-squared analysis (i.e., Fisher ${ }^{2}$ ). The $R^{2}$ was computed as the squared Pearson correlation between the positions at the selected proportions of the MT and the position at movement end (e.g., $R^{2} @ 75 \% \%_{\mathrm{MT}}$ ). Likewise, the Fisher variable was calculated as the Fisher $r$-to$Z$-transformed versions of the same correlations, and Fisher $^{2}$ was the square of the Fisher variable. These trajectory-based measures were also assessed in both the primary and secondary movement axes (e.g., Fisher ${ }_{\text {Prim }}$ and Fisher $_{\text {Sec }}$ ). These variables were analyzed both to help assess their own relative validity and to validate the frequency analysis that followed.

The last, and critical, set of dependent variables assessed was evaluated in the frequency domain. Acceleration trajectories were recorded with the accelerometer in the primary and secondary movement axes. To isolate trajectory discontinuities, a 5th-order polynomial was fit to each trajectory, and the residuals were converted into proportional power spectra (pPower) with a frequency resolution of $1.56 \mathrm{~Hz}$ (see Fig. 1). A 5th-order polynomial was chosen for the fit because the acceleration traces of discrete aiming movements exhibit four bends (i.e., one centered around movement initiation, one centered around peak acceleration, one centered around peak deceleration, and one centered around movement end). The detrending of the acceleration trace allowed for the direct comparison of discontinuities across conditions, irrespective of differences in the MTs. The power spectra were calculated by first applying a Hanning truncation window to the residuals of the polynomial fit, and the FFT was computed using the $\mathrm{fft}()$ function in MATLAB using a transform length of 128 samples (i.e., to capture a movement duration up to $640 \mathrm{~ms}$ ). The application of the Hanning window ensured that the data substrate slowly tapered to zero at the ends, which reduces the presence artifacts in the output of the FFT (see Randall, 2008; Warner, 1998). It also served the purpose of minimizing the influence of any accelerometer artifacts present at the start or end of the movement. The power spectra were computed from the FFT output, and the proportional power was assessed at each frequency as a percentage of the total power up to the Nyquist frequency (i.e., up to $100 \mathrm{~Hz}$ ). For the purposes of the analysis, pPower was assessed across seven frequencies ranging from 1.56 up to $10.94 \mathrm{~Hz}$.

All dependent variables were statistically evaluated using repeated measures analyses of variance (ANOVAs). The analyses of the movement-end-based variables, along with the analyses of JScore and PV, were all structured as 2 Vision Condition $(\mathrm{FV}, \mathrm{NV}) \times 3$ Target Amplitude $(27,30,33 \mathrm{~cm})$ repeated measures ANOVAs. The analyses of the trajectory-based variables associated with multiple movement proportions (i.e., Variability, $R^{2}$, Fisher, and Fisher ${ }^{2}$ ) were assessed with the same first two factors and with the additional factor Movement Proportion $(25 \%, 50 \%$, or $75 \%$ of MT). In addition, the analysis of the variability measure included a fourth level of Movement Proportion — namely, $100 \%$ of the MT. The repeated measures ANOVA completed on the frequency-based measure (i.e., pPower) had a 2 Vision Condition $(\mathrm{FV}, \mathrm{NV}) \times 3$ Target Amplitude $(27,30,33 \mathrm{~cm}) \times 7$ Frequency $(1.56,3.12$, $4.68,6.25,7.81,9.37,10.93 \mathrm{~Hz})$ design. This range of frequencies was chosen because the minimum time required to use visual information for online control has been reported to be approximately 100 ms (i.e., $10 \mathrm{~Hz}$; Carlton, 1992; Saunders \& Knill, 2003; Zelaznik et al., 1983). Across all analyses, when the assumption of sphericity was violated, GreenhouseGeisser-corrected degrees of freedom were used to assess significance. All corrected degrees of freedom are reported to the first decimal place. Significant main effects and interactions were further assessed using a simple main effects approach and Bonferroni-corrected multiple comparisons. Because Vision Condition was the most theoretically relevant factor, the decomposition of the significant interactions was completed to examine the differences across rather than within levels of Vision Condition. For these comparisons, the Bonferroni correction was applied on the basis of the number of comparisons made rather than the total number of possible comparisons. The statistical analysis was completed using R (R Development Core Team, 2014), and the ANOVAs were computed with the EZ package (Lawrence, 2013). All effect sizes reported represent the generalized eta squared (i.e., $\eta_{\mathrm{G}}{ }^{2}$; see Bakeman, 2005; Olejnik \& Algina, 2003). In the case of analyses yielding significant main effects and significant interactions, only the interactions were further analyzed and interpreted.

\section{Results}

\section{Movement end dependent variables}

The MT analyses resulted in main effects of vision condition $\left[F(1,9)=6.10, p=.036, \eta_{\mathrm{G}}{ }^{2}=.009\right]$ and target amplitude 
Fig. 1 An example of the data reduction process. a The raw acceleration profile for the primary axis of movement. $\mathbf{b}$ The best fitting 5 th-order polynomial of this profile. $\mathbf{c}$ The substrate for the frequency analysis. The residuals of the polynomial fit were multiplied by a Hanning truncation window. d The proportional power spectra of these preprocessed residuals
A

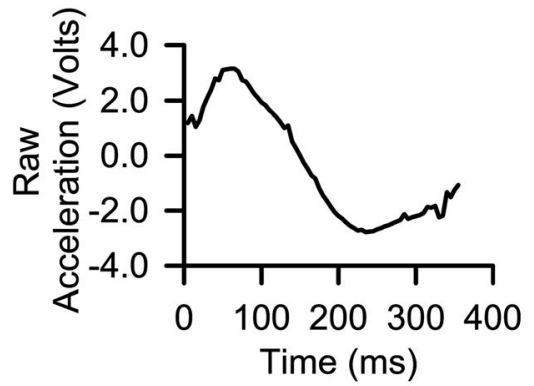

C

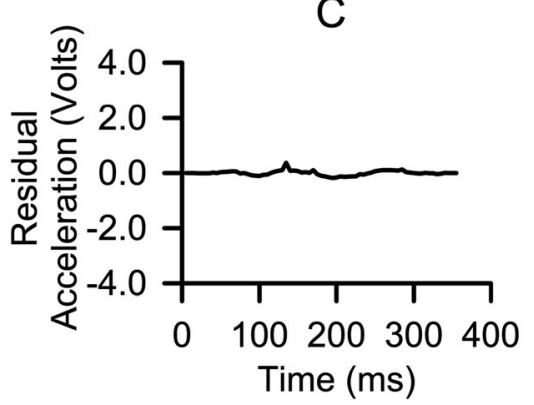

B
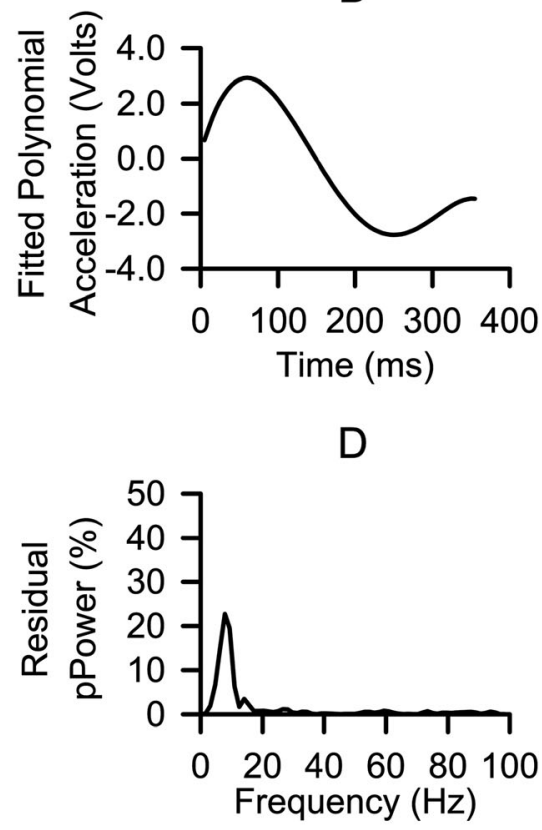

$\left[F(2,18)=33.4, p<.001, \eta_{\mathrm{G}}{ }^{2}=.117\right]$. Movements completed in the presence of vision (i.e., FV) resulted in significantly longer MTs (i.e., $405 \mathrm{~ms}, S D=46$ ) than did movements completed without vision (i.e., NV; $396 \mathrm{~ms}, S D=50$ ). Posthoc analyses of the main effect of target amplitude indicated that movements to farther targets resulted in significantly longer MTs (i.e., $27 \mathrm{~cm}$ : $378 \mathrm{~ms}, S D=49$; $30 \mathrm{~cm}$ : $401 \mathrm{~ms}, S D=48$; and $33 \mathrm{~cm}$ : $421 \mathrm{~ms}, S D=51)$. Significant differences were observed between each level of target amplitude (all $p \mathrm{~s}<.008$ ).

The TPV analyses yielded only a significant main effect of target amplitude $\left[F(1.7,15.6)=12.56, p=.003, \eta_{\mathrm{G}}{ }^{2}=.056\right]$. Farther target amplitudes resulted in longer TPV values (i.e., $27 \mathrm{~cm}$ : $151 \mathrm{~ms}, S D=21 ; 30 \mathrm{~cm}: 157 \mathrm{~ms}, S D=25 ; 33 \mathrm{~cm}$ : $161 \mathrm{~ms} S D=33$ ). Post-hoc analyses revealed significant differences between each of these levels of target amplitude (all $p s<.033$ ).

The TAPV analyses resulted only in a significant main effect of target amplitude $[F(1.5,13.7)=21.50, p<.001$, $\left.\eta_{\mathrm{G}}{ }^{2}=.120\right]$. Longer target amplitudes resulted in longer TAPV values (i.e., $27 \mathrm{~cm}: 232 \mathrm{~ms}, S D=34 ; 30 \mathrm{~cm}: 249 \mathrm{~ms}$, $S D=31 ; 33 \mathrm{~cm}: 259 \mathrm{~ms}, S D=26$ ). Post-hoc analysis revealed that all target amplitude levels were significantly different from one another (all $p$ s < .037). In contrast to the TAPV findings, pTAPV resulted in no significant main effects or interactions $\left(F \mathrm{~s}<1.3, p \mathrm{~s}>.280, \eta_{\mathrm{G}}{ }^{2} \mathrm{~s}<.007\right)$ (grand mean $=62 \%, S D=4)$.

The assessment of $\mathrm{CE}_{\text {Prim }}$ (i.e., the constant error in the primary movement axis) resulted in a main effect of vision condition $\left[F(1,9)=64.64, p<.001, \eta_{\mathrm{G}}{ }^{2}=.246\right]$. Positive and negative values indicated target overshoots and undershoots, respectively. Movements completed without vision (i.e., NV) resulted in significantly shortened movement amplitudes (i.e.,
$-0.4 \mathrm{~mm}, S D=2.7$ ), as compared to movements completed with vision (i.e., $3.4 \mathrm{~mm}, S D=1.7$ ). The analysis of $C E_{\mathrm{Sec}}$ (i.e., constant error in the secondary movement axis) yielded main effects of both vision condition $[F(1,9)=32.95, p<$ $\left..001, \eta_{\mathrm{G}}{ }^{2}=.104\right]$ and target amplitude $[F(1.2,10.4)=6.81, p$ $\left.=.014, \eta_{\mathrm{G}}{ }^{2}=.177\right]$. Note that positive and negative values for $\mathrm{CE}_{\mathrm{Sec}}$ indicated movement endpoints that were farther or closer to the body, respectively, relative to the target. Movements completed without vision (i.e., NV) resulted in endpoints that were farther than the target away from the body (i.e., $6.2 \mathrm{~mm}$, $S D=3.6)$ than did movements completed with vision (i.e., $\mathrm{FV} ; 3.9 \mathrm{~mm}, S D=2.5$ ). Post-hoc analyses of the main effect of target amplitude resulted in a significant difference between the $33-\mathrm{cm}$ (i.e., $2.8 \mathrm{~mm}, S D=3.6$ ) and the $30-\mathrm{cm}$ (i.e., $5.7 \mathrm{~mm}, S D=2.9 ; p=.002$ ) target amplitudes. Additionally, the comparison between the $33-\mathrm{cm}$ and $27-\mathrm{cm}$ (i.e., $6.6 \mathrm{~mm}$, $S D=3.9$ ) target amplitudes approached statistical significance $(p=.053)$.

The $\mathrm{VE}_{\text {Prim }}$ analyses resulted in a significant main effect of vision condition $\left[F(1,9)=7.05, p=.026, \eta_{\mathrm{G}}{ }^{2}=.097\right]$. Movements completed without vision resulted in larger endpoint distributions (i.e., $8.1 \mathrm{~mm}, S D=0.8$ ) than did movements completed with vision (i.e., $7.1 \mathrm{~mm}, S D=1.3$ ). Conversely, the analysis of $\mathrm{VE}_{\mathrm{Sec}}$ did not result in any main effects or interactions $\left(F \mathrm{~s}<1.09, p \mathrm{~s}>.358, \eta_{\mathrm{G}}{ }^{2} \mathrm{~s}=.152\right)$ (grand mean $=5.2 \mathrm{~mm}, S D=0.6$ ).

\section{Spatio-temporal dependent variable}

The PV analyses yielded a significant main effect of target amplitude $\left[F(1.1,10.2)=70.84, p<.001, \eta_{\mathrm{G}}{ }^{2}=.082\right]$. Larger target amplitudes led to larger PV values (i.e., $27 \mathrm{~cm}: 1.4 \mathrm{~m} / \mathrm{s}$, 
$S D=0.3 ; 30 \mathrm{~cm}: 1.5 \mathrm{~m} / \mathrm{s}, S D=0.3 ; 33 \mathrm{~cm}: 1.6 \mathrm{~m} / \mathrm{s}, S D=0.3$ ). Post-hoc analyses of this main effect identified significant differences between all target amplitude levels ( $p s<.005$ ).

\section{Trajectory-based dependent variables}

Because the effects of MT proportion (i.e., $25 \%, 50 \%$, and $\left.75 \%_{\mathrm{MT}}\right)$ in the trajectory-based dependent variables that included it were expected and theoretically trivial, significant main effects and interactions involving MT proportion were not subjected to post-hoc comparisons across levels of MT proportion.

The jerk score analyses in the primary movement axis (i.e., JScore $\left._{\text {Prim }}\right)$ resulted in a main effect of target amplitude $[F(1.3$, $\left.11.4)=27.25, p<.001, \eta_{\mathrm{G}}{ }^{2}=.152\right]$. JScore Prim $_{\text {increased with }}$ increases in target amplitude (i.e., $27 \mathrm{~cm}: 1,466, S D=148$; $30 \mathrm{~cm}: 1,551, S D=122$; and $33 \mathrm{~cm}: 1,601, S D=112$ ). Posthoc analyses revealed significant differences between all levels of target amplitude (all $p$ s <.008). The analyses of JScore $_{\text {Sec }}$ yielded a significant main effect of target amplitude $\left[F(2,18)=17.34, p<.001, \eta_{\mathrm{G}}{ }^{2}=.161\right]$. JScore Sec $_{\text {increased }}$ with increases in target amplitude (i.e., $27 \mathrm{~cm}: 1,535, S D=$ 255; $30 \mathrm{~cm}: 1,692, S D=272$; and $33 \mathrm{~cm}: 1,819, S D=278$ ). Post-hoc analyses revealed significant differences between all levels ( $p$ s <.005), except for the comparison between the 27 $\mathrm{cm}$ and 30-cm target amplitudes $(p=.075)$.

The analyses of the variability across movements in the primary movement axis (i.e., Variability Prim $_{\text {) }}$ resulted in a main effect for movement proportion $[F(1.1,14.3)=42.33$, $\left.p<.001, \eta_{\mathrm{G}}{ }^{2}=.044\right]$. Variability ${ }_{\text {Prim }}$ changed across the movement proportions (i.e., $25 \%_{\mathrm{MT}}: 16.12 \mathrm{~mm}, S D=4.99 \mathrm{~mm}$; $50 \%_{\mathrm{MT}}: 23.54 \mathrm{~mm}, S D=5.97 ; 75 \%_{\mathrm{MT}}: 12.41 \mathrm{~mm}, S D=$ $\left.2.41 ; 100 \%_{\mathrm{MT}}: 7.61 \mathrm{~mm}, S D=0.93\right)$. The main effect of target amplitude neared conventional levels of statistical significance $\left[F(2,18)=3.11, p=.069, \eta_{\mathrm{G}}{ }^{2}=.044\right]$. The Target Amplitude $\times$ Movement Proportion interaction $[F(2.7,24.5)=$ 2.82, $\left.p=.065, \eta_{\mathrm{G}}{ }^{2}=.029\right]$ and the Vision Condition $\times$ Movement Proportion interaction $[F(1.3,12.1)=3.55, p=$ $\left..075, \eta_{\mathrm{G}}{ }^{2}=.024\right]$ also neared conventional levels of statistical significance. The Variability ${ }_{S e c}$ analyses yielded significant main effects of target amplitude $[F(2,18)=7.51, p=.004$, $\left.\eta_{\mathrm{G}}{ }^{2}=.048\right]$ and movement proportion $[F(1.3,11.4)=18.71$, $\left.p<.001, \eta_{\mathrm{G}}{ }^{2}=.273\right]$. Variability $y_{\text {Prim }}$ tended to increase with increasing target amplitude (i.e., $27 \mathrm{~cm}: 4.8 \mathrm{~mm}, S D=1.1$; $30 \mathrm{~cm}: 5.4 \mathrm{~mm}, S D=1.3$; and $33 \mathrm{~cm}: 5.6 \mathrm{~mm}, S D=1.2$ ). Posthoc analyses of the target amplitude effect indicated that the $27-\mathrm{cm}$ target exhibited significantly lower variability than did the 30 - and $33-\mathrm{cm}$ targets $(p \mathrm{~s}<.04)$. Across movement proportions, Variability $\mathrm{Sec}_{\mathrm{s}}$ increased and then decreased (i.e., $25 \%_{\mathrm{MT}}: 3.8, S D=1.5 ; 50 \%_{\mathrm{MT}}: 6.4, S D=1.9 ; 75 \%_{\mathrm{MT}}$ : $5.7, S D=0.8 ;$ and $100 \%: 5.2, S D=0.6$ ).

Analysis of the $R_{\text {Prim }}^{2}$ data resulted in main effects of vision condition $\left[F(1,9)=10.24, p=.011, \eta_{\mathrm{G}}{ }^{2}=.067\right]$ and movement proportion $\left[F(1.1,9.5)=64.45, p<.001, \eta_{\mathrm{G}}{ }^{2}=\right.$ 408]. Trials completed in the presence of visual feedback resulted in significantly lower $R^{2}$ values (i.e., $.16, S D=.05$ ), as compared to trials completed in the absence of vision (i.e., $.25, S D=.09) . R_{\text {Prim }}^{2}$ increased with increases in movement proportion (i.e., $25 \%_{\mathrm{MT}}: .11, S D=.03 ; 50 \%_{\mathrm{MT}}: .13, S D=$ $.05 ; 75 \%$ MT: $: 40, S D=.12$ ). The $R^{2}$ Sec analyses resulted only in a main effect of movement proportion $[F(2,18)=120.43, p$ $\left.<.001, \eta_{\mathrm{G}}{ }^{2}=.605\right]: R^{2}$ Sec increased with increasing movement proportion (i.e., $25 \%_{\mathrm{MT}}: .13, S D=.07 ; 50 \%_{\mathrm{MT}}: .35, S D=$ $.13 ; 75 \%_{\mathrm{MT}}$. $\left.67, S D=.14\right)$.

The assessment of the Fisher $Z$-transformed correlations in the primary movement axis (i.e., Fisher $_{\text {Prim }}$ ) resulted in a main effect of movement proportion $[F(1.2,10.6))=123.38, p<$ $\left..001, \eta_{\mathrm{G}}{ }^{2}=.418\right]$ and an interaction between vision condition, target amplitude, and movement proportion $[F(4,36)=3.71$, $\left.p<.013, \eta_{\mathrm{G}}{ }^{2}=.010\right]$. This three-way interaction was further analyzed by running separate 2 Vision Condition $\times 3$ Movement Proportion repeated measures ANOVAs for each of the three target amplitudes. Although all three analyses yielded significant main effects of movement proportion [all $\left.F_{\mathrm{s}}(2,18)>38, p \mathrm{~s}<.001, \eta_{\mathrm{G}}{ }^{2}>.350\right]$, only the 33 -cm target amplitude resulted in a significant Vision Condition $\times$ Movement Proportion interaction $[F(1.3,11.5)=4.53, p=$ $\left..049, \eta_{\mathrm{G}}{ }^{2}=.028\right]$. Post-hoc analyses of this interaction, however, indicated that no significant differences existed between vision conditions across proportions for the $33-\mathrm{cm}$ target amplitude (all $p$ s $>.191$ ). The analysis of Fisher Sec $_{\text {resulted only }}$ in a main effect of movement proportion $[F(2,18)=125.79$, $\left.p<.001, \eta_{\mathrm{G}}{ }^{2}=.583\right]$; Fisher ${ }_{\mathrm{Sec}}$ increased with increasing movement proportion (i.e., $25 \%_{\mathrm{MT}}: 0.26, S D=0.20$; $50 \%_{\mathrm{MT}}: 0.67, S D=0.21 ; 75 \%_{\mathrm{MT}}: 1.24, S D=0.26$ ).

Squared Fisher $Z$ values in the primary movement axis (i.e., Fisher $_{\text {Prim }}^{2}$ ) resulted in significant main effects of vision condition $\left[F(1,9)=14.16, p=.004, \eta_{\mathrm{G}}{ }^{2}=.047\right]$ and movement proportion $\left[F(1.0,9.2)=33.55, p<.001, \eta_{\mathrm{G}}{ }^{2}=.309\right]$. Also, the interaction between vision condition and movement proportion neared conventional levels of statistical significance $\left[F(1.1,9.9)=3.91, p=.074, \eta_{\mathrm{G}}{ }^{2}=.016\right]$. Trials completed in the presence of visual feedback resulted in significantly lower Fisher $^{2}{ }_{\text {Prim }}$ values (i.e., $0.25, S D=0.13$ ) than did trials completed in the absence of vision (i.e., $0.44, S D=0.19$ ). Our Fisher $^{2}$ Sec analysis resulted in a main effect of movement proportion $\left[F(1.1,10.3)=58.36, p<.001, \eta_{\mathrm{G}}{ }^{2}=.540\right]$ : Fisher $^{2}$ Sec increased with increasing levels of movement proportion (i.e., $25 \%_{\mathrm{MT}}: 0.15, S D=0.10 ; 50 \%_{\mathrm{MT}}: 0.56, S D=0.26 ; 75 \%_{\mathrm{MT}}$ : $1.68, S D=0.64)$.

\section{Frequency-based dependent variables}

Because significant main effects of frequency were expected and theoretically trivial, these significant main effects were not subjected to post-hoc comparisons. The evaluation of the 
proportional power spectra of the acceleration traces in the primary movement axis (i.e., pPower $_{\text {Prim }}$ ) resulted in significant main effects for target amplitude $[F(2,18)=5.84, p=$ $\left..011, \eta_{\mathrm{G}}^{2}=.009\right]$ and frequency $[F(1.4,12.9)=20.84, p<$ $\left..001, \eta_{\mathrm{G}}{ }^{2}=.630\right]$. Also, a significant Vision Condition $\times$ Frequency interaction was found $[F(2.0,17.7)=5.54, p<$ $\left..014, \eta_{\mathrm{G}}{ }^{2}=.014\right]$. Overall, proportional power increased with increasing target amplitude (i.e., $27 \mathrm{~cm}: 8.8 \%, S D=1.2$; $30 \mathrm{~cm}: 9.3 \%, S D=1.1 ; 33 \mathrm{~cm}: 9.7 \%, S D=1.1)$. Post-hoc analyses of the target amplitude effect revealed a significant difference in proportional power between the target amplitudes of 27 and $33 \mathrm{~cm}$ only $(p=.039)$. The Vision Condition $\times$ Frequency interaction was further evaluated through post-hoc comparisons between vision conditions across the range of frequencies. This analysis resulted in a significant difference in proportional power between FV and $\mathrm{NV}$ at the $4.68-\mathrm{Hz}$ frequency level only $(p<.001)$ (see Fig. 2). That is, in the presence of online visual information, the proportional power at the $4.68-\mathrm{Hz}$ level (i.e., $M=12.7 \%, S D=$ 5.8) was significantly greater than the proportional power attained when vision was unavailable during action (i.e., $M=10.9 \%, S D=5.8$ ).

The assessment of proportional power in the secondary movement axis (i.e., pPower $_{\mathrm{Sec}}$ ) yielded significant main effects of vision condition $\left[F(1,9)=7.99, p=.020, \eta_{\mathrm{G}}{ }^{2}=.007\right]$ and frequency $\left[F(1.6,14.3)=23.76, p<.001, \eta_{\mathrm{G}}{ }^{2}=.606\right]$. Additionally, we observed a significant three-way interaction between vision condition, target amplitude, and frequency $\left[F(3.9,34.7)=3.70, p=.014, \eta_{\mathrm{G}}{ }^{2}=.018\right]$. This three-way interaction was first assessed by completing a series of 2 Vision Condition $\times 7$ Frequency repeated measures ANOVAs - that is, one for each of the three target amplitudes.

First, regarding the $27-\mathrm{cm}$ target amplitude, significant main effects were found for vision condition $[F(1,9)=6.50$,

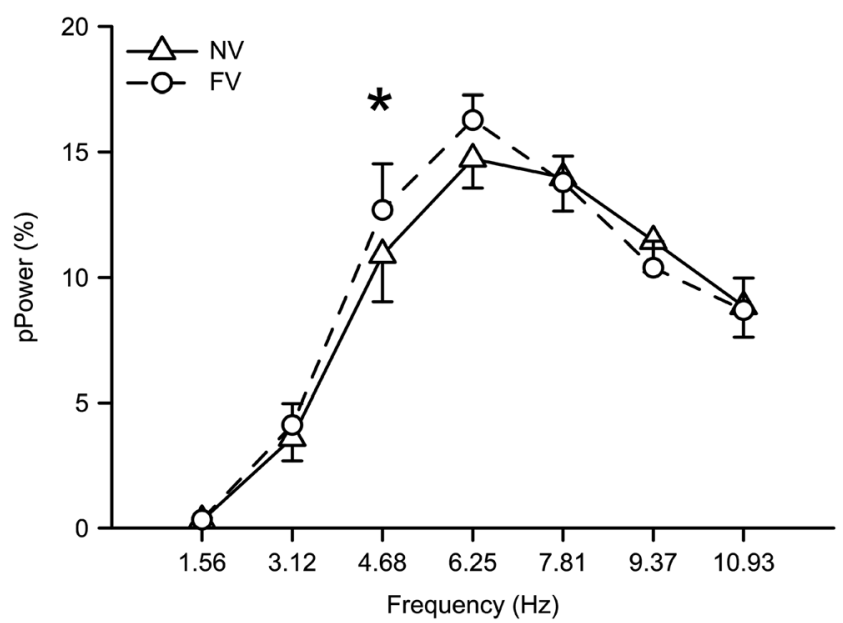

Fig. 2 Proportional power across frequencies for the two vision conditions in the primary movement axis. The FV condition exhibited significantly greater proportional power in the $4.68-\mathrm{Hz}$ frequency. Error bars represent one between-subjects SEM $\left.p<.031, \eta_{\mathrm{G}}^{2}=.011\right]$ and frequency $[F(1.5,13.2)=18.99, p<$ $\left..001, \eta_{\mathrm{G}}{ }^{2}=.612\right]$. In the presence of vision, the proportional power across the range of frequencies examined was significantly greater (i.e., $M=7.6 \%, S D=1.3$ ) than when vision was unavailable during action (i.e., $M=6.9 \%, S D=1.4$ ). Second, regarding the $30-\mathrm{cm}$ target amplitude, significant effects were found for the main effect of frequency $[F(1.8,16.1)=23.14$, $\left.p<.001, \eta_{\mathrm{G}}{ }^{2}=.620\right]$ and the interaction between vision condition and frequency $\left[F(2.0,18.4)=8.07, p=.003, \eta_{\mathrm{G}}{ }^{2}=\right.$ $.050]$. Post-hoc comparisons between vision conditions across levels of frequency indicated nonsignificant differences, although the comparisons made at $4.68 \mathrm{~Hz}(\mathrm{FV}: 10.6 \%, S D=$ 4.7; NV: $8.1 \%, S D=3.9)$ and $6.25 \mathrm{~Hz}(\mathrm{FV}: 14.5 \%, S D=4.6$; $\mathrm{NV}: 11.8 \%, S D=3.3$ ) neared statistical significance (i.e., $p \mathrm{~s}$ of .07 and .056 , respectively). Finally, regarding the $33-\mathrm{cm}$ target amplitude, a main effect of frequency was found $\left[F(2.0,18.1)=20.61, p<.001, \eta_{\mathrm{G}}^{2}=.591\right]$. The main effect of vision condition also neared statistical significance $\left[F(1,9)=4.90, p=.054, \eta_{\mathrm{G}}{ }^{2}=.008\right]$.

\section{Discussion}

One goal of the present study was to evaluate the ability of frequency analyses to identify changes in performance attributable to online visual feedback utilization during discrete reaching movements. This goal was achieved by comparing participants' reaching performance under conditions wherein online visual information was either available or not available. Differences between vision conditions were observed in both the primary and secondary movement axes. In the primary movement axis, increases in pPower were observed at the $4.68-\mathrm{Hz}$ frequency. In the secondary axis, no specific frequency was identified; however, pPower increased in general in the FV condition relative to the NV condition. Such effects in pPower potentially represented differences in online visual feedback utilization. These differences were corroborated by $\mathrm{FV}$-versus-NV differences with conventional measures of online control.

The secondary goal of the present study was to compare existing measures of online control in terms of their sensitivities to changes in feedback utilization brought about by manipulations of vision (i.e., FV vs. NV condition.). To this end, an analysis was undertaken of the effect sizes involving the Vision Condition factor. Additionally, the effect sizes associated with theoretically driven individual comparisons were also examined to identify the most sensitive individual measures. Ultimately, correlation-based measures such as $R^{2}$ and Fisher $^{2}$ yielded the highest individual effect sizes. The pPower analyses resulted in the highest within-trial effect sizes and represented a viable alternative. 


\section{Conventional measures of online feedback utilization}

Overall, the analysis of conventional measures of online control resulted in significant FV-versus-NV differences. These findings therefore helped to corroborate any differences found in the frequency analyses. First, considering the movement end temporal variables, a significant main effect of vision condition was found for MT. Longer MTs were observed for movements with as compared to without online visual feedback (e.g., Carson, Chua, Elliott, \& Goodman, 1990). Such an effect can theoretically be attributed in part to the temporal costs associated with the use of feedback (see Fitts, 1954). Indeed, when utilizing targets with relatively high indices of difficulty (i.e., indices of difficulty above 3.58 bits; see Wallace \& Newell, 1983), FV-versus-NV performance differences may be more confidently associated with visual feedback utilization. As such, in the present study we employed targets approximately $1 \mathrm{~cm}$ in width at distances ranging from 27 to $33 \mathrm{~cm}$, yielding indices of difficulty from 5.75 to 6.04 bits. As a result, we have a reasonable level of confidence that the presence of vision during the movement (i.e., online feedback use) explains the observed differences in the investigated measures of online control. Unlike previous research, however, we found no significant differences for TAPV or pTAPV (cf. Elliott et al., 1991), although the mean difference between vision conditions was in the expected direction for TAPV (i.e., $\mathrm{FV}=251 \mathrm{~ms}$ vs. NV = $243 \mathrm{~ms}$ ). Notably, other studies have also failed to detect TAPV differences between FV and NV conditions (e.g., Heath et al., 2004).

Second, considering the movement end spatial variables, significant effects involving vision condition were found for $\mathrm{CE}_{\text {Prim }}, \mathrm{CE}_{\mathrm{Sec}}$, and $\mathrm{VE}_{\text {Prim. }}$. The analysis of $\mathrm{CE}_{\text {Prim }}$ resulted in significant undershooting in the $\mathrm{NV}$ condition relative to the FV condition. Additionally, reaching movements were significantly more accurate in the secondary movement axis (i.e., $\mathrm{CE}_{\mathrm{Sec}}$ ) in the $\mathrm{FV}$ than in the $\mathrm{NV}$ condition. Also, significantly smaller VEPrim was observed in the FV than in the NV condition. Each of these patterns of data have been reported previously for comparable reaching conditions (e.g., Heath, 2005).

Third, the analysis of the trajectory-based measures resulted in significant effects involving vision condition for the $R_{\text {Prim }}^{2}$, Fisher ${ }^{2}{ }_{\text {Prim }}$, and Variability Prim variables. Overall, performance in the presence of online visual feedback (i.e., the $\mathrm{FV}$ condition) resulted in lower $R_{\text {Prim }}^{2}$ and Fisher ${ }^{2}$ Prim values than when online visual feedback was not available (i.e., in the $\mathrm{NV}$ condition). These main effects were in line with previous findings differentiating FV from NV conditions (e.g., Heath, 2005; Khan, Lawrence, Franks, \& Buckolz, 2004; Tremblay, Hansen, Kennedy, \& Cheng, 2013; cf. Bernier et al., 2006). As such, these analyses of $R_{\text {Prim }}^{2}$ and Fisher ${ }^{2}$ Prim have provided evidence of greater alterations of ongoing trajectories in the presence of online visual feedback than during performance in the absence of online visual feedback.
Ultimately, these analyses of conventional measures of feedback-related processes (i.e., online control) in the present study have provided converging evidence that participants made use of the visual information available for feedbackrelated processes during reaches. Therefore, significant FVversus-NV differences in the conventional measures of online control could be confidently associated with differential use of feedback processes.

\section{Frequency-based measurement of online feedback utilization}

Through our analysis of contemporary measures of online feedback utilization, it appeared that participants were indeed making use of online visual information in the FV condition relative to the NV condition. Thus, a closer look at the differences in the pPower spectra from the frequency analysis was warranted. Vision condition significantly influenced the pPower spectra for both the primary and secondary movement axes. In the primary axis, significantly greater pPower was observed at the $4.68-\mathrm{Hz}$ frequency in the presence of online visual feedback (i.e., the FV condition), as compared to performance without feedback available (i.e., the NV condition). Likewise, the secondary movement axis yielded a similar FVversus-NV difference, although a specific frequency of influence could not be identified. Because the FV-versus-NV differences in performance were associated with specific changes in the pPower spectra in the primary movement axis, it is possible that these changes could be associated with differential use of online visual feedback. Thus, analysis in the frequency domain appears to be sensitive to changes in performance brought about by differences in online feedback utilization in discrete aiming movements.

The critical frequency of $4.68 \mathrm{~Hz}$ can be associated as first glance with a feedback iteration time of $214 \mathrm{~ms}$. That is, because the changes in performance brought about through changes in online feedback utilization were found to be related through a specific common frequency (i.e., $4.68 \mathrm{~Hz}$ ), the use of feedback may exert its influence at the associated timebased rate. This frequency of $4.68 \mathrm{~Hz}$ and its associated iteration rate (i.e., $214 \mathrm{~ms}$ per cycle) are actually congruent with the hypothesized influence of feedback at $10 \mathrm{~Hz}$ (i.e., $100 \mathrm{~ms}$ per cycle; e.g., Saunders \& Knill, 2005). The apparent discrepancy can be alleviated if one acknowledges the possibility that in the absence of discrete secondary accelerations, corrective processes may be characterized by graded accelerations or decelerations rather than discrete acceleration-deceleration pairs (see Wisleder \& Dounskaia, 2007). Also, because pPower represents trajectories in terms of sine and cosine waveforms, the $4.68-\mathrm{Hz}$ frequency represents the rate of acceleration-deceleration pairs. Therefore, the difference in pPower at $4.68 \mathrm{~Hz}$ can be taken as evidence for the presence of graded adjustments of acceleration or deceleration that 
Table 1 Effect sizes associated with significant effects for measures not including the Movement Proportion factor

\begin{tabular}{lllllllllll}
\hline Effect & \multicolumn{1}{l}{ Dependent Variable } & & & & \\
\cline { 2 - 8 } & MT & TTPV & TAPV & pTAPV & CE $_{\text {Prim }}$ & CE $_{\text {Sec }}$ & VE $_{\text {Prim }}$ & VE $_{\text {Sec }}$ & JScore $_{\text {Prim }}$ & JScore $_{\text {Sec }}$ \\
\hline Vision Condition & $.009^{*}$ & - & - & - & $.246^{* * *}$ & $.104^{* * *}$ & $.097^{*}$ & - & - \\
Target Amplitude & $.117^{* * * *}$ & $.056^{* *}$ & $.120^{* * *}$ & - & - & $.177^{*}$ & - & - & $.152^{* * *}$ & $.161^{* * * *}$ \\
Vision Condition $\times$ Target Amplitude & - & - & - & - & - & - & - & - & - \\
\hline
\end{tabular}

Effect size reported $=$ generalized eta squared (i.e., $\eta_{\mathrm{G}}{ }^{2}$ ); “_” indicates $p \geq .05 .{ }^{*} p<.05 .{ }^{* *} p<.01 .{ }^{* * *} p<.001$

could be occurring at twice this rate (i.e., $9.36 \mathrm{~Hz}$ ). This higher frequency estimate of the latency of online feedback utilization results in an iteration time of approximately $107 \mathrm{~ms}$. Such a value is consistent with currently accepted corrective reaction times to visual perturbations (i.e., $100 \mathrm{~ms}$; see Brenner \& Smeets, 1997; Carlton, 1992; Saunders \& Knill, 2005; Zelaznik, Hawkins, \& Kisselburgh, 1983). Therefore, the level of frequency that was associated with performance differences was consistent with online visual feedback utilization latencies.

Although it may have also appeared that pPower scaled proportionally with movement amplitude, a strict association between movement amplitude and pPower was not universally found. That is, despite the fact that the NV condition undershot the FV condition in the primary movement axis and overshot the FV condition in the secondary movement axis, significant differences between the FV as compared to the NV condition were observed in the analyses of both axes. ${ }^{1}$ Nonetheless, the specificity of the frequencies responsible for differentiating between the vision conditions was more clearly defined for the primary movement axis (i.e., FV > $\mathrm{NV}$ at $4.68 \mathrm{~Hz}$ only) than for the secondary movement axis (i.e., $\mathrm{FV}>\mathrm{NV}$ main effect).

Whereas in the present study we manipulated target amplitude, the target width remained constant across targets. The absence of significant interactions between target amplitude and vision condition, at least for the primary movement axis, indicated a relatively robust pattern across the range of target amplitudes examined. Thus, further work explicitly manipulating accuracy demands (i.e., target widths) or employing a greater range of amplitudes and different movement directions

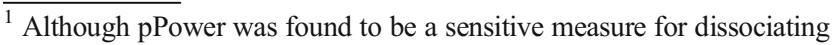
performance across the FV and NV conditions, it was theoretically possible that changes in MT were driving the observed effects. In order to test this possibility, a supplementary analysis was completed wherein between vision condition $t$ tests for MT and pPower $_{\text {Prim }}$ at $4.68 \mathrm{~Hz}$ were completed for each target. If the MT differences were indeed driving the pPower effects, then a comparable pattern of differences between vision conditions would be expected. Regarding the MT data, a significant FV-versus-NV difference was observed for the $30-\mathrm{cm}$ target only (i.e., $p=.009)$. Conversely, significant FV-versus-NV differences in pPower $_{\text {Prim }}$ at $4.68 \mathrm{~Hz}$ were observed for both the $27-\mathrm{cm}$ and $30-\mathrm{cm}$ targets (i.e., $p \mathrm{~s}<.015$ ). Thus, although MT and pPower may be related measures, they are distinct indicators of online control.
}

could prove useful to provide support for this association between pPower and feedback utilization with greater confidence. That is, in the present study we could not explicitly evaluate the influence of large changes in MT, accuracy demands, movement amplitude, or direction on pPower, which should be addressed in future investigations. Furthermore, because the frequencies evaluated in the analysis of pPower were dependent on the collection rate and the MTs (i.e., $200 \mathrm{~Hz} / 128$ samples $=1.56-\mathrm{Hz}$ increments), the preciseness of the estimates of iteration times should be taken with a certain degree of caution (see Warner, 1998). Ultimately, this frequency-based analysis remains subject to the same criticism as conventional measures of online control, because they are all indirect or implied measure of online control. It must be recognized that a direct evaluation of the underlying nature of online feedback utilization would require additional neurophysiological measurements, which is beyond the scope of the present study. Nevertheless, frequency-based analyses were found to be sensitive to changes in performance associated with online feedback utilization (i.e., performance differences across FV vs. NV) in a manner that may also identify the latency at which online visual feedback has been utilized.

\section{A comparison of methods: Effect sizes}

The secondary goal of the present study was undertaken through an analysis of the effect sizes associated with the significant effects across all measures differentiating the FV from the NV condition. Although it is generally good practice to run power analyses in order to set appropriate sample sizes for investigations, the exploratory nature of the present study did not afford a predicted relevant effect size on which to base such an analysis. Instead, a post-hoc review of the achieved effect sizes across all variables was undertaken. Specifically, a comparison across the measures' effect sizes was used to evaluate their relative sensitivities to changes in online feedback utilization. Regarding all significant effects able to differentiate between the levels of the Vision Condition factor (i.e., FV, $\mathrm{NV}), \mathrm{VE}_{\text {Prim }}, R_{\text {Prim }}^{2}$, and Fisher ${ }_{\text {Prim }}^{2}$ yielded in the highest $\eta_{\mathrm{G}}{ }^{2}$ values (i.e., .097, .067, and .047, respectively; see Tables 1 and 2). The significant effects in the pPower analysis resulted in $\eta_{\mathrm{G}}{ }^{2}$ values that were in the same range (i.e., .014 and .018, 
Table 2 Effect sizes for the significant effects associated with measures that include the Movement Proportion factor

\begin{tabular}{|c|c|c|c|c|c|c|c|c|}
\hline \multirow[t]{2}{*}{ Effect } & \multicolumn{8}{|c|}{ Dependent Variable } \\
\hline & $R_{\text {Prim }}^{2}$ & $R_{\mathrm{Sec}}^{2}$ & Fisher $_{\text {Prim }}$ & Fisher $_{\text {Sec }}$ & Fisher ${ }^{2}$ Prim & Fisher $^{2} \mathrm{Sec}$ & Variability $_{\text {Prim }}$ & Variabilitysec $_{\mathrm{S}}$ \\
\hline Vision Condition & $.067^{*}$ & - & - & - & $.047^{* *}$ & - & - & - \\
\hline Target Amplitude & - & - & - & - & - & - & $.044^{* * * *}$ & $.048^{* *}$ \\
\hline Movement Proportion & $.408^{* * * *}$ & $.605^{* * *}$ & $.418^{* * * * *}$ & $.583^{* * * *}$ & $.309^{* * *}$ & $.521^{* * * *}$ & $.521^{* * * *}$ & $.273^{* * *}$ \\
\hline Vision Condition $\times$ Target Amplitude & - & - & - & - & - & - & - & - \\
\hline Vision Condition $\times$ Movement Proportion & - & - & - & - & - & - & - & - \\
\hline Target Amplitude $\times$ Movement Proportion & - & - & - & - & - & - & - & - \\
\hline $\begin{array}{l}\text { Vision Condition } \times \text { Target Amplitude } \times \text { Movement } \\
\text { Proportion }\end{array}$ & - & - & $.010^{*}$ & - & - & - & - & - \\
\hline
\end{tabular}

Effect size reported $=$ generalized eta squared (i.e., $\eta_{\mathrm{G}}{ }^{2}$ ); “-” indicates $p \geq .05 .{ }^{*} p<.05 .{ }^{* *} p<.01 .{ }^{* * * *} p<.001$

respectively; see Table 3) as that of Fisher ${ }_{\text {Prim }}$ (i.e., .010), and greater than that of MT (i.e., .009). Although the effect sizes associated with pPower were not the largest effect sizes attained, they were certainly in the range of currently utilized measures.

The observed pattern of greater effect sizes for $\mathrm{CE}$ and $\mathrm{VE}$ relative to pTAPV and trajectory-based variability measures (see Tables 1 and 2) has been previously reported by Elliott and Hansen (2010). Elliott and Hansen did not, however, report equivalently high effect sizes for the $R^{2}$ analysis. Indeed, they did not report a significant effect involving vision condition for the analysis of $R^{2}$. The key difference between the work of Elliott and Hansen and the present study could have stemmed from differences in offline (i.e., trial-to-trial) changes in performance. That is, because Elliott and Hansen utilized only one target (cf. the three target amplitudes in the present study) and "blocked" their vision conditions (cf. the randomized vision conditions in the present study), participants may have planned their movements differently across conditions. Additionally, Elliott and Hansen computed their $R^{2}$-type analyses from fewer trials per condition (i.e., 20) and through correlations at the occurrences of kinematic markers in the primary movement axis (i.e., peak acceleration, peak velocity, and peak deceleration; see also Heath, 2005). In contrast, in the present study we utilized a greater number of trials (i.e., 30) and normalized MT proportions (i.e., $25 \%_{\mathrm{MT}}, 50 \%_{\mathrm{MT}}$, and $75 \%$ MT; see also Tremblay et al., 2013). Thus, it may be that such analyses are more sensitive regarding assessments of the percentage of MT (cf. Heath, 2005) or that a greater number of trials should be utilized (i.e., 30; see also Heath, 2005).

In addition to the general comparison of the effect sizes of the significant effects involving vision condition, the effect sizes associated with the most theoretically driven specific comparisons were assessed separately (see Table 4). This analysis was completed to determine the measure that was most sensitive to vision manipulations, and which specific comparison could be most informative regarding evidence for the presence of online control. The comparisons considered included VE $E_{\text {Prim }}$, MT, pTAPV, $R_{\text {Prim }}^{2}$, Fisher ${ }^{2}$ Prim, $R_{\text {Prim }}^{2}$ $75 \%_{\mathrm{MT}}$, Fisher ${ }_{\text {Prim } @ 75 \%}$, and pPower @ $4.68 \mathrm{~Hz}$ for both the primary and secondary movement axes. On the basis of a direct comparison of the effect sizes associated with these variables, the largest effect size was associated with Fisher $^{2}$ Prim @ $75 \%_{\mathrm{MT}}$, followed by $R_{\text {Prim }}^{2} @ 75 \%_{\mathrm{MT}}$ and $\mathrm{VE}_{\text {Prim. }}$. The effect sizes associated pPower in the primary and secondary movement axes resulted in intermediate effect sizes that were, however, larger than the effect sizes associated

Table 3 Effect sizes for the significant effects associated with the frequency-based dependent variables

\begin{tabular}{lll}
\hline Effect & \multicolumn{1}{c}{ Dependent Variable } \\
\cline { 2 - 3 } & pPower $_{\text {Prim }}$ & pPower $_{\text {Sec }}$ \\
\hline Vision Condition & - & $.007^{*}$ \\
Target Amplitude & $.009^{*}$ & - \\
Frequency & $.630^{* * *}$ & $.606^{* * *}$ \\
Vision Condition $\times$ Target Amplitude & - & - \\
Vision Condition $\times$ Frequency & $.014^{*}$ & - \\
Target Amplitude $\times$ Frequency & - & - \\
Vision Condition $\times$ Target Amplitude $\times$ Frequency & - & $.018^{*}$ \\
\hline
\end{tabular}

Effect size reported $=$ generalized eta squared (i.e., $\eta_{\mathrm{G}}{ }^{2}$ ). “-” indicates $p \geq .05 .{ }^{*} p<.05 .{ }^{* * * *} p<.001$ 
Table 4 Effect sizes of individual comparisons between vision conditions for dependent variables exhibiting significant effects involving vision condition

\begin{tabular}{|c|c|c|c|c|c|c|c|}
\hline \multirow[t]{2}{*}{ Effect } & \multicolumn{7}{|c|}{ Dependent Variable } \\
\hline & MT & TAPV & $\mathrm{VE}_{\text {Prim }}$ & $R_{\text {Prim }}^{2} @ 75 \%_{\mathrm{MT}}$ & Fisher $^{2}{ }_{\text {Prim }} @ 75 \%_{\mathrm{MT}}$ & pPower $_{\text {Prim }} @ 4.68 \mathrm{~Hz}$ & pPower $_{\mathrm{Sec}} @ 4.68 \mathrm{~Hz}$ \\
\hline Effect size & .009 & .017 & .097 & .131 & .166 & .025 & .032 \\
\hline Rank & 7 & 6 & 3 & 2 & 1 & 5 & 4 \\
\hline
\end{tabular}

Effect size reported $=$ generalized eta squared (i.e., $\eta_{\mathrm{G}}{ }^{2}$ )

with the other within-trial measures - namely, MT, TAPV, and pTAPV. Ultimately, these findings indicate that the most sensitive current measure of online visuomotor control, as evidenced by the present assessment of effect sizes, is Fisher ${ }^{2}{ }_{\text {Prim }}$ @ $75 \%$ MT. However, the trial-by-trial nature of Fisher ${ }^{2}$ Prim is also sensitive to offline changes in performance, which are not necessarily attributable to changes in online feedback utilization. For example, an individual may choose to change the prioritization of speed versus accuracy based on performance on a trial-to-trial basis (e.g., de Grosbois, Heath, \& Tremblay, 2015). That is, individuals may move their finger into the vicinity of the target at different rates across trials. As such, the form of the movement trajectory could vary significantly from trial to trial, irrespective of changes in online feedback utilization. Such changes in performance could come about as a function of practice. That is, the relative symmetry of reaching movements to a target can change over a series of trials with the concurrent visual information that is available (e.g., Khan et al., 1998). Overall, this type of between-trial variability could theoretically induce false positives in the $R^{2}$ and Fisher ${ }^{2}$ measures by lowering the predictability across trials of the relation between the distance traveled at earlier movement proportions and the distance traveled at movement end. The within-trial nature of the removal of the unique 5thorder polynomial trend from each of the acceleration profiles, followed by the frequency analyses forwarded in the present study, could theoretically circumvent the above-mentioned potential contamination of offline processing in measures of online feedback utilization. That is, the specificity of the differences in the frequency domain could theoretically identify changes in feedback utilization irrespective of offline, trial-bytrial changes in performance.

\section{Conclusion}

Overall, we found the evaluation of Fisher ${ }^{2}$ Prim at $75 \%$ of the movement time to be the best measure for dissociating performance between conditions wherein online visual feedback was either available or withheld. However, the between-trial nature of the measure could have increased the susceptibility of the measure to offline changes in performance. In contrast, analyses in the frequency domain at $4.68 \mathrm{~Hz}$ for the acceleration trajectories could be a more sensitive within-trial measure of online control and may be more robust to trial-by-trial changes in performance.

Although the use of sensory feedback during goal-directed actions is ubiquitous, the measurement of online feedback utilization has proven challenging. In the present study, we applied analyses in the frequency domain, through proportional power spectra, to identify the presence of feedback processes and the latency at which they were taking place during discrete reaching movements. These analyses were successful in differentiating performance in the presence versus absence of online visual feedback, while also confirming a potential minimum iterative rate of approximately $107 \mathrm{~ms}$. Ultimately, the application of this frequency-based analysis to reaching trajectories provides an additional avenue to identifying and quantifying online feedback utilization.

Author note This research was supported by the Natural Sciences and Engineering Research Council of Canada, the Canada Foundation for Innovation, and the Ontario Research Fund.

\section{References}

Bakeman, R. (2005). Recommended effect size statistics for repeated measures designs. Behavior Research Methods, 37, 379-384. doi: 10.3758/BF03192707

Bernier, P.-M., Chua, R., Franks, I. M., \& Khan, M. A. (2006). Determinants of offline processing of visual information for the control of reaching movements. Journal of Motor Behavior, 38, 331-338. doi:10.3200/JMBR.38.5.331-338

Bracewell, R. N. (1986). The Fourier transform and its applications. New York: McGraw-Hill.

Brenner, E., \& Smeets, J. B. J. (1997). Fast responses of the human hand to changes in target position. Journal of Motor Behavior, 29, 297 310. doi:10.1080/00222899709600017

Carlton, L. (1992). Visual processing time and the control of movement. In L. Proteau \& D. Elliott (Eds.), Advances in psychology: Vision and motor control (pp. 3-32). Amsterdam: North-Holland.

Carson, R. G., Chua, R., Elliott, D., \& Goodman, D. (1990). The contribution of vision to asymmetries in manual aiming. Neuropsychologia, 28, 1215-1220. doi:10.1016/0028-3932(90) 90056-T

Cheng, D. T., Luis, M., \& Tremblay, L. (2008). Randomizing visual feedback in manual aiming: Reminiscence of the previous trial 
condition and prior knowledge of feedback availability. Experimental Brain Research, 189, 403-410. doi:10.1007/s00221008-1436-3

Craik, K. J. W. (1947). Theory of the human operator in control systems: I. The operator as an engineering system. British Journal of Psychology, 38, 56-61.

Cressman, E. K., Cameron, B. D., Lam, M. Y., Franks, I. M., \& Chua, R. (2010). Movement duration does not affect automatic online control. Human Movement Science, 29, 871-881. doi:10.1016/j.humov. 2010.07.001

Crossman, E. R. F. W., \& Goodeve, P. J. (1983). Feedback control of hand- movement and Fitts' law. Quarterly Journal of Experimental Psychology, 35A, 251-278. doi:10.1080/14640748308402133

de Grosbois, J., Heath, M., \& Tremblay, L. (2015). Augmented feedback influences upper limb reaching movement times but does not explain violations of Fitts' Law. Frontiers in Psychology, 6, 800. doi: $10.3389 /$ fpsyg.2015.00800

R Development Core Team (2014). R: A language and environment for statistical computing. Vienna, Austria: R Foundation for Statistical Computing. Retrieved from www.R-project.org/

Dounskaia, N., Wisleder, D., \& Johnson, T. (2005). Influence of biomechanical factors on substructure of pointing movements. Experimental Brain Research, 164, 505-516. doi:10.1007/s00221005-2271-4

Elliott, D., \& Allard, F. (1985). The utilization of visual feedback information during rapid pointing movements. Quarterly Journal of Experimental Psychology, 37A, 407-425. doi:10.1080/ 14640748508400942

Elliott, D., Binsted, G., \& Heath, M. (1999). The control of goal-directed limb movements: Correcting errors in the trajectory. Human Movement Science, 18, 121-136. doi:10.1016/S0167-9457(99) 00004-4

Elliott, D., Carson, R., Goodman, D., \& Chua, R. (1991). Discrete vs. continuous visual control of manual aiming. Human Movement Science, 10, 393-418. doi:10.1016/0167-9457(91)90013-N

Elliott, D., \& Hansen, S. (2010). Visual regulation of manual aiming: A comparison of methods. Behavior Research Methods, 42, 10871095. doi:10.3758/BRM.42.4.1087

Elliott, D., Hansen, S., Grierson, L. E. M., Lyons, J., Bennett, S. J., \& Hayes, S. J. (2010). Goal-directed aiming: Two components but multiple processes. Psychological Bulletin, 136, 1023-1044. doi: 10.1037/a0020958

Elliott, D., \& Madalena, J. (1987). The influence of premovement visual information on manual aiming. Quarterly Journal of Experimental Psychology, 39A, 541-559. doi:10.1080/14640748708401802

Fitts, P. (1954). The information capacity of the human motor system in controlling the amplitude of movement. Journal of Experimental Psychology, 47, 381-391. doi:10.1037/h0055392

Flash, T., \& Hogan, N. (1985). The coordination of arm movements: An experimentally confirmed mathematical model. Journal of Neuroscience, 5, 1688-1703.

Heath, M. (2005). Role of limb and target vision in the online control of memory-guided reaches. Motor Control, 9, 281-311.

Heath, M., Westwood, D. A., \& Binsted, G. (2004). The control of memory-guided reaching movements in peripersonal space. Motor Control, 8, 76-106.

Howarth, C. I., Beggs, W. D. A., \& Bowden, J. (1971). The relationship between speed and accuracy of movement aimed at a target. Acta Psychologica, 35, 207-218. doi:10.1016/0001-6918(71)90022-9

Keele, S. W., \& Posner, M. I. (1968). Processing of visual feedback in rapid movements. Journal of Experimental Psychology, 77, 155158. doi: $10.1037 / \mathrm{h} 0025754$

Khan, M. A., Franks, I. M., Elliott, D., Lawrence, G. P., Chua, R., Bernier, P., ... Weeks, D. J. (2006). Inferring online and offline processing of visual feedback in target-directed movements from kinematic data.
Neuroscience and Biobehavioral Reviews, 30, 1106-1121. doi:10. 1016/j.neubiorev.2006.05.002

Khan, M. A., Franks, I. M., \& Goodman, D. (1998). The effect of practice on the control of rapid aiming movements: Evidence for an interdependency between programming and feedback processing. Quarterly Journal of Experimental Psychology, 51A, 425-443. doi:10.1080/713755756

Khan, M. A., Lawrence, G., Fourkas, A., Franks, I. M., Elliott, D., \& Pembroke, S. (2003). Online versus offline processing of visual feedback in the control of movement amplitude. Acta Psychologica, 113, 83-97. doi:10.1016/S0001-6918(02)00156-7

Khan, M. A., Lawrence, G. P., Franks, I. M., \& Buckolz, E. (2004). The utilization of visual feedback from peripheral and central vision in the control of direction. Experimental Brain Research, 158, 241251. doi:10.1007/s00221-004-1897-y

Lawrence, M. A. (2013). ez: Easy analysis and visualization of factorial experiments ( $\mathrm{R}$ package version 4.2-2). Retrieved from http:// CRAN.R-project.org/package $=\mathrm{ez}$

Messier, J., \& Kalaska, J. F. (1999). Comparison of variability of initial kinematics and endpoints of reaching movements. Experimental Brain Research, 125, 139-152. doi:10.1007/s002210050669

Meyer, D. E., Abrams, R. A., Kornblum, S., Wright, C. E., \& Smith, J. E. (1988). Optimality in human motor performance: Ideal control of rapid aimed movements. Psychological Review, 95, 340-370. doi: 10.1037/0033-295X.95.3.340

Olejnik, S., \& Algina, J. (2003). Generalized eta and omega squared statistics: Measures of effect size for some common research designs. Psychological Methods, 8, 434-447. doi:10.1037/1082989X.8.4.434

Plamondon, R., \& Alimi, A. M. (1997). Speed/accuracy trade-offs in target-directed movements. Behavioral and Brain Sciences, 20, 279-303. doi:10.1017/S0140525X97001441

Proteau, L., Roujoula, A., \& Messier, J. (2009). Evidence for continuous processing of visual information in a manual video-aiming task. Journal of Motor Behavior, 41, 219-231. doi:10.3200/JMBR.41.3. 219-231

Randall, R. B. (2008). Spectral analysis and correlation. In D. Havelock, S. Kuwano, \& M. Vorlander (Eds.), Handbook of signal processing in acoustics (pp. 33-52). New York: Springer.

Saunders, J. A., \& Knill, D. C. (2003). Humans use continuous visual feedback from the hand to control fast reaching movements. Experimental Brain Research, 152, 341-352. doi:10.1007/s00221003-1525-2

Saunders, J. A., \& Knill, D. C. (2005). Humans use continuous visual feedback from the hand to control both the direction and distance of pointing movements. Experimental Brain Research, 162, 458-473. doi:10.1007/s00221-004-2064-1

Tremblay, L., Hansen, S., Kennedy, A., \& Cheng, D. T. (2013). The utility of vision during action: multiple visuomotor processes? Journal of Motor Behavior, 45, 91-99. doi:10.1080/00222895. 2012.747483

Tresilian, J. R., Stelmach, G. E., \& Adler, C. H. (1997). Stability of reachto-grasp movement patterns in Parkinson's disease. Brain, 120, 2093-2111. doi:10.1093/brain/120.11.2093

van Donkelaar, P., \& Franks, I. M. (1991). Preprogramming vs. on-line control in simple movement sequences. Acta Psychologica, 77, 119. doi:10.1016/0001-6918(91)90061-4

van Galen, G. P., \& Schomaker, L. R. B. (1992). Fitts' law as a low-pass filter effect of muscle stiffness. Human Movement Science, 11, 1121. doi:10.1016/0167-9457(92)90046-E

Van Galen, G. P., Van Doorn, R. R., \& Schomaker, L. R. (1990). Effects of motor programming on the power spectral density function of finger and wrist movements. Journal of Experimental Psychology. Human Perception and Performance, 16, 755-765. doi:10.1037/ 0096-1523.16.4.755 
Walker, N., Philbin, D., Worden, A., \& Smelcer, J. B. (1997). A program for parsing mouse movements into component submovements. Behavior Research Methods, Instruments, \& Computers, 29, 456460. doi:10.3758/BF03200599

Wallace, S. A., \& Newell, K. M. (1983). Visual control of discrete aiming movements. Quarterly Journal of Experimental Psychology, 35A, 311-321. doi:10.1080/14640748308402136

Warner, R. M. (1998). Spectral analysis of time series data. New York: Guilford Press.
Wisleder, D., \& Dounskaia, N. (2007). The role of different submovement types during pointing to a target. Experimental Brain Research, 176, 132-149. doi:10.1007/s00221-006-0603-7

Woodworth, R. S. (1899). The accuracy of voluntary movement. Psychological Review: Monograph Supplements, 3(3), 1-114.

Zelaznik, H. N., Hawkins, B., \& Kisselburgh, L. (1983). Rapid visual feedback processing in single-aiming movements. Journal of Motor Behavior, 15, 217-236. 